\title{
Perforación gástrica secundaria a resucitación cardiopulmonar: reporte de un caso
}

\author{
Daniela Grant-Alpízar*, Wagner Ramírez-Quesada, Pablo Coste-Murillo, Ricardo Barahona-García y \\ Gerardo Avendaño-Alvarado \\ Servicio de Gastroenterología, Hospital Rafael Ángel Calderón Guardia, San José, Costa Rica
}

\section{Resumen}

Las complicaciones abdominales como la distensión gástrica y los desgarros mucosos secundarios a la resucitacióñcardiopulmonar $(R C P)$ no son infrecuentes, sin embargo, la perforación gástrica es muy inusual y su evidencia endoscópica anecdótica. Se presenta el caso de un varón de 58 años con perforación gástrica y sangrado digestivo alto, en el cuâl se brindó RCP prolongada por muerte súbita extrahospitalaria.

Palabras clave: Perforación gástrica. Resucitación cardiopulmonar. Complicaciones abdominales. Sangrado digestivo ălto.

\section{Gastric perforation secondary to cardiopulmonary resuscitation: A case report}

\section{Abstract}

Abdominal complications such as gastric distention and mucosal tears secondary to cardiopulmonary resuscitation (GPR) are not uncommon, however, gastric perforation is very unusual, and its endoscopic evidence is anecdotical. A case of a 58-year-old man with gastric perforation and upper gastrointestinal bleeding is presented, in which prolonged CPR performed due to out-of-hospital sudden death.

Key words: Gastric perforation. Cardiopulmonary resuscitation. Abdominal complications. Upper gastrointestinal bleedin̄g

\section{Presentación de caso}

Varón de 58 años que presenta muerte súbita extrahospitalaria y es inmediatamente atendido por una enfermera presencial, quien aplica protocolo de soporte vital básico durante 30 minutos, hasta que llega el personal de apoyo. Al presentarse el equipo de atención prehospitalaria, se le documenta una fibrilación ventricular, la cual se revierte evidenciando ritmo sinusal con pulso.
Es trasladado al servicio de emergencias más cêrcano, en donde se decide realizar intubación endotra-

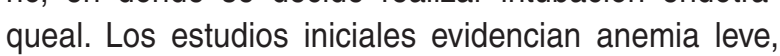
una radiografía de tórax con un neumotórax derech̆o y un electrocardiograma con elevación del ST en ceara anterior (Fig. 1). Se le colocó una sonda de tórax \$ se realizó intervención arterial coronaria percutáneaccon colocación de dos stents coronarios e inicio de triple terapia antiplaquetaria. Ameritó vasopresores durănte el procedimiento. 


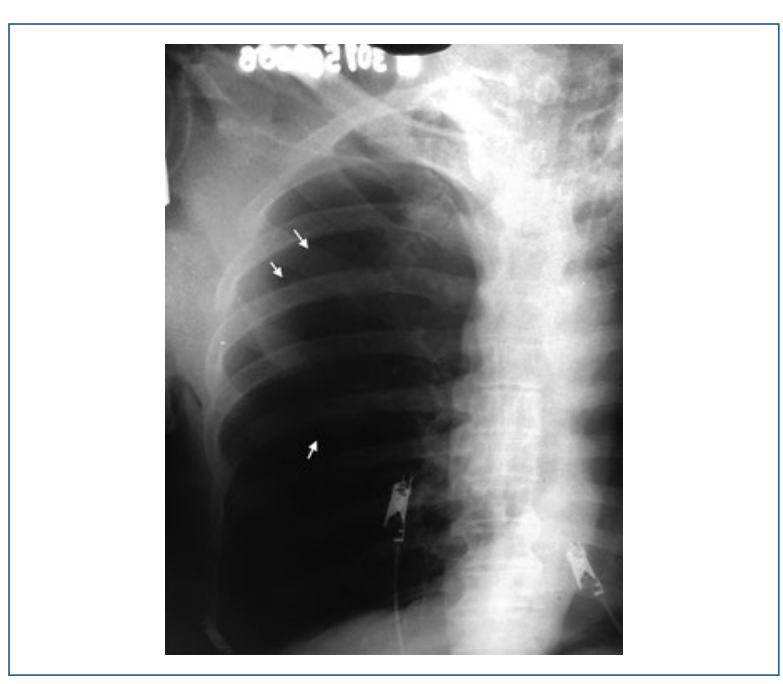

Figura 1. Neumotórax derecho (se señala la línea de la pleura visceral).

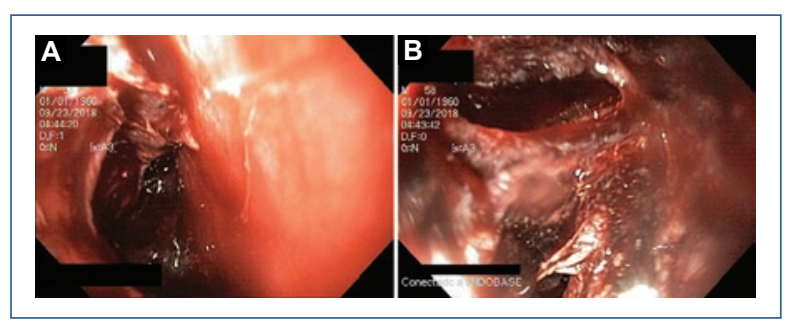

Figura 2. A y B: perforación gástrica de $3 \mathrm{~cm}$ a lo largo de curvatura menor, bajo unión esofagogástrica.

A su traslado a la Unidad de Cuidados Intensivos, evidenció salida de sangre por la sonda nasogástrica y aumento del requerimiento de vasopresores. Se realizó una endoscopia digestiva alta de emergencia, documentando una rotura gástrica extensa en curvatura menor, con sangrado profuso e incontrolable con métodos endoscópicos (Fig. 2). Pese a la terapia con hemoderivados, durante su traslado a la sala de operaciones presentó parada cardiorrespiratoria sin respuesta a maniobras.

\section{Discusión}

El riesgo de trauma iatrogénico relacionado con la resucitación cardiopulmonar (RCP) se presenta incluso con su adecuada ejecución, evidenciándose lesiones abdominales (dilatación gástrica, laceraciones viscerales, desgarros mucosos, etc.) hasta en un $30.8 \%$ de las autopsias $^{1,2}$. A pesar de la frecuente ocurrencia de estas complicaciones, la perforación gástrica traumâtica secundaria a RCP es una complicación rara que conlleva alta mortalidad si no se identifica a tiempo $0^{1,3}$ Se presume que la principal causa de perforación gástricica traumática es la distensión gástrica debido a una ventilación artificial inadecuada y al aumento de las@presiones intragástricas durante las compresiones toráci$\mathrm{cas}^{4,5}$. En este sentido, la realización de RCP Ppor espectadores (56\%), el uso de bolsa mascarilla (21\%) y una vía aérea difícil (21\%) son los principales factores relacionados con esta complicación $n^{4,5}$. Es importante considerar su ocurrencia cuando se observa neumồperitoneo o distensión abdominal súbita ${ }^{6}$.

Los casos reportados de rotura gástrica relacionădos con RCP han resultado en perforación a lo largo de la curvatura menor del estómago, usualmente adyacênte a la unión gastroesofágica. Esta es la porción menos elástica del estómago debido a que tiene menos pliegues mucosos y mayor fijación por el ligamênto hepatogástrico ${ }^{4,7}$.

La mortalidad en el diagnóstico temprano depende principalmente de la patología inicial. La laparotomía de emergencia es el tratamiento más frecuente, pero debe considerarse la posibilidad de manejo conservador en casos seleccionados ${ }^{4,6}$.

En este paciente, a diferencia de lo que describe la literatura, la presentación y principal complicación no fue el hemo/neumoperitoneo o la distensión abdominal, sino un sangrado digestivo alto en relación con ưna rotura no tributaria a cierre endoscópico y a la antiagregación farmacológica.

\section{Financiamiento}

No se recibió patrocinio de ningún tipo para la reạlización de este artículo.

\section{Declaración de conflicto de intereses}

Los autores declaran no tener ningún conflictê de intereses.

\section{Responsabilidades éticas}

Protección de personas y animales. Los auteres declaran que para esta investigación no se han realizado experimentos en seres humanos ni en animales.

Confidencialidad de los datos. Los autores declaran que han seguido los protocolos de su centrō de trabajo sobre la publicación de datos de pacientes. 
Derecho a la privacidad y consentimiento informado. Los autores declaran que en este artículo no aparecen datos de pacientes.

\section{Bibliografía}

1. Buschmann CT, Tsokos M. Frequent and rare complications of resuscitation attempts. Intensive Care Med. 2009;35(3):397-404.

2. Krischer JP, Fine EG, Davis JH, et al. Complications of cardiac resuscitation. Chest. 1987;92(2):287-91.
3. Khan A, Merrett N, Selvendran S. Stomach perforation post cardiopulmonary resuscitation-A case report. Int J Surg Case Rep. 2017;40:43-6.

4. Spoormans I, van Hoorenbeeck K, Balliu L, et al. Gastric perforation after cardiopulmonary resuscitation: Review of the literature. Resuscitation. 2010;81(3):272-80.

5. Afacan MA, Colak S, Gunes H, et al. An unusual complication of cardiopulmonary resuscitation: Stomach perforation. Am J Emerg $\mathrm{T}$ Med. 2014;32(9):1149.e1-1149.e3.

6. Jalali SM, Emami-Razavi H, Mansouri A. Gastric perforation after cardiopulmonary resuscitation. Am J Emerg Med. 2012;30(9):2091.e1-2091.e22.

7. Arai $Y$, Honjo S, Shimizu S, et al. Traumatic gastric perforation associated with cardiopulmonary resuscitation: A case report. Yonago Acta Med. 2017;60(3):204-8 